\title{
An Investigation into the Enhancement of Fingermarks in Blood on Paper with Genipin and Lawsone
}

\author{
Paula Thomas, Kevin Farrugia * \\ School of Contemporary Sciences, Division of Environment and Forensic Sciences, \\ University of Abertay, Dundee, DD1 1HG, UK
}

\section{* Corresponding Author:}

School of Contemporary Sciences

Division of Environment and Forensic Sciences

University of Abertay

Dundee DD1 1HG

United Kingdom

tel: $+44(0) 1382308689$

fax: +44 (0) 1382308663

kevin.farrugia@abertay.ac.uk 


\begin{abstract}
The abilities of two natural products, genipin and lawsone, to enhance blood contaminated fingermarks on papers of various porosities and colour were investigated and compared to the routinely used amino acid reagents, ninhydrin and 1,8-diazafluoren-9-one (DFO).

Fingermarks in blood were deposited as a split depletion series on various paper types and colours for ageing periods of 6 weeks, 4 weeks, 2 weeks and 1 week before enhancement. The developed marks were observed under different lighting conditions, recorded and graded by way of attributing quantitative data to each series.

Results indicated that while genipin showed some potential as a reagent for the enhancement of latent fingermarks, it displayed no suitability for the enhancement of fingermarks in blood on paper. Lawsone also failed to successfully enhance either type of fingermark. Upon comparison of the results with those of ninhydrin and DFO it was found that ninhydrin displayed the highest success rate of development of these marks.
\end{abstract}

Keywords: fingermarks, ninhydrin, DFO, genipin, lawsone, blood enhancement 


\section{INTRODUCTION}

Chemical reagents such as ninhydrin and DFO (1,8-diazafluoren-9-one) are routinely used for the enhancement of latent fingermarks on porous surfaces such as papers [1]. These reagents react with the amino acids in fingermark secretions to form new compounds that display colour and/or fluorescent properties. Amino acids are also present in blood, thus fingermarks contaminated in blood may be enhanced with such amino acid reagents. Two naturally sourced plant products, genipin and lawsone, have recently been identified as having the potential to chemically enhance latent fingermarks [2-4].

\section{Genipin and Lawsone}

Genipin is a natural product that can be extracted from the gardenia fruit and is the hydrolytic product of geniposide. Genipin is a colourless substance that reacts with amino acids to produce a blue coloured complex with fluorescent properties optimised at an illumination of 590nm and viewed with a barrier above 630nm [2, 3]. Lawsone, 2-hydroxy-1,4naphthoquinone, is sourced from the leaves of the Lawsonia inermis plant and is used in Henna dyes. Lawsone belongs to a group of compounds known as naphthoquinones and recent studies $[4,5]$, reported the reaction of lawsone with amino acids in latent fingermarks to produce a purple-brown colour that also exhibits fluorescent properties with a maximum intensity of about $640 \mathrm{~nm}$ with an excitation wavelength of $590 \mathrm{~nm}$.

\section{Ninhydrin and DFO}

Ninhydrin (2,2-dihydroxy-1,3-indanedione) is an amino acid reagent that reacts with amino acids to form a non-fluorescent purple product, known as Ruhemanm's purple. The reaction is accelerated through the use of heat and humidity. Beside the enhancement of latents and fingermarks in blood, several studies have discussed the successful enhancement of footwear marks in blood with ninhydrin. A study [6] of the enhancement of footwear impressions in blood on various surfaces demonstrated that ninhydrin can yield good quality marks displaying clear pattern details. Ninhydrin was also successful in enhancing footwear impressions in blood on paper that had been exposed to rain for over three weeks [7] as well as footwear marks in blood on different types of fabric [8]. The successful use of ninhydrin and DFO for the treatment of stains in blood followed by successful subsequent DNA typing has also been demonstrated [9-10]. 
Similar to ninhydrin, DFO reacts with amino acids to produce a highly fluorescent, red coloured product which requires the use of an alternative light source for visualisation with a recommended excitation wavelength of $430-580 \mathrm{~nm}$. In addition, when compared to ninhydrin, DFO appears to demonstrate a higher sensitivity for the development of latent fingermarks. The Manual of Fingerprint Development Techniques by the UK Home Office Centre for Applied Science and Technology (CAST, formerly the Home Office Scientific Development Branch) indicates that ninhydrin can be used as part of a sequential process and if used after DFO in sequential treatment of the same fingermark impression, additional detail could be developed [1]. In contrast, little or no further development occurs if DFO is used after ninhydrin. Under optimum conditions, DFO will develop more latent fingermark impressions than ninhydrin, however the latter develops visible prints whereas DFO may also require an additional fluorescence observation step for visualisation.

A review of amino acid staining techniques for latent fingermarks on porous surfaces revealed that ninhydrin was the predominant reagent for the successful development of latent fingermarks [11]. Figure 1 illustrates the chemical structures of ninhydrin, DFO, genipin and lawsone. To date no studies have been reported which compare the suitability of genipin and lawsone to ninhydrin and DFO for the enhancement of fingermarks in blood on paper.<smiles>O=C1c2ccccc2C(=O)C1(O)O</smiles>

(a)

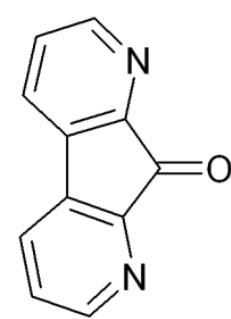

(b)<smiles>COC(=O)C1=COC(O)C2C(CO)=CCC12</smiles>

(c)<smiles>O=C1C=C(O)C(=O)c2ccccc21</smiles>

(d)

Figure 1 - The chemical structures of (a) ninhydrin; (b) DFO; (c) genipin and (d) lawsone 


\section{Materials and Methods}

\section{Paper substrates and ageing periods}

Eight papers of different porosities and colours were selected as potential substrates, all purchased locally (table 1). Each paper type was planted with a diminishing series of 10 fingermarks in blood and allowed to age at room temperature on bench surfaces in the laboratory for the following time intervals: 1 week, 2 weeks, 4 weeks and 6 weeks. The groups of samples were then enhanced with each reagent after their ageing period.

Table 1 - Types of paper used in the study

\begin{tabular}{|c|c|}
\hline Type of Paper & Supplier/ Manufacturer \\
\hline 80 gsm white & Tesco Stores Ltd \\
\hline 80 gsm pink & PDQ Print Services \\
\hline 80 gsm green & PDQ Print Services \\
\hline 100 gsm white & Daler-Rowney \\
\hline 120 gsm white & Paper State \\
\hline Magazine (glossy) & Adsa Stores Ltd \\
\hline Leaflet (glossy) & Domino's Pizza Group Ltd \\
\hline Newspaper & Metro Newspaper \\
\hline
\end{tabular}

\section{Diminishing series}

The researcher's finger was dabbed in laked horse blood that had been poured into a petridish. The finger was then pressed twice onto a clean, blue, chemical free laboratory paper towel to remove the excess blood before a diminishing series was planted on the various paper substrates. The series consisted of 10 fingermarks that ranged from visible to barely visible to latent. The comparison and evaluation of enhancement techniques was performed using a split print method [12]. 
Positive and negative controls were performed for each substrate. The positive control samples consisted of latent fingermarks being planted on the papers without the presence of blood. The negative control samples contained no fingermarks (latent or in blood) in order to ensure that any potential reaction was not due to the reagent reacting with the paper.

\section{Photography and Quasar}

Each depletion series was photographed before and after enhancement using a Nikon D5000 digital SLR camera with a $55 \mathrm{~mm}$ lens. The camera was set to its manual setting at a focal length of F5.6 and positioned at a $90^{\circ}$ angle to the sample. This was aided by the use of a Kaiser camera stand containing lights positioned at $45^{\circ}$ angles to the samples.

The quasar used during this study was a Mason Vectron Quasar 2000/30 connected to an Integrated Rapid Imaging System (IRIS). Table 2 ilustrates the excitation and viewing filters used for each chemical. The wavelength ranges represented in table 2 show the $1 \%$ cut-on and cut-off points respectively. Other light sources may use wavelengths representing the $50 \%$ point or the peak wavelength. Although ninhydrin does not fluoresce, the use of a violet/blue excitation filter may improve the contrast with the background $[1,13]$. Any fluorescence observed was photographed using the IRIS camera and computer software.

Table 2 - Excitation and viewing filters for chemicals used in the study

\begin{tabular}{|c|c|c|c|c|}
\hline Chemical & $\begin{array}{c}\text { Excitation } \\
\text { Wavelength/nm }\end{array}$ & $\begin{array}{c}\text { Excitation } \\
\text { Filter }\end{array}$ & $\begin{array}{c}\text { Viewing } \\
\text { Wavelength/nm }\end{array}$ & Viewing Filter \\
\hline Ninhydrin & $350-469$ & Violet/Blue & 476 & Yellow \\
\hline DFO & $503-587$ & Green/Yellow & 593 & Red \\
\hline DFO & $491-548$ & Green & 549 & Orange \\
\hline Genipin & $503-587$ & Green/Yellow & 593 & Red \\
\hline Lawsone & $503-587$ & Green/Yellow & 593 & Red \\
\hline
\end{tabular}

\section{Chemical reagents and treatment processes}

The formulations in this study are those utilised by the United States Secret Service which include petroleum ether rather than HFE solvents as recommended by Ramotowski if 
flammability is not a major concern [14]. All treated items were then analysed under the IRIS for fluorescence as indicated in table 2.

\section{Ninhydrin [14]}

Ninhydrin (6g, Sigma) was completely dissolved in ethanol (50mL, Fisher) using a magnetic stirrer. Petroleum ether (950mL, Fisher) was then added with further stirring.

The ninhydrin solution was poured into a shallow glass dish and the paper sample to be treated was immersed in the solution for a few seconds. The samples were then allowed to air dry before being placed in a heat and humidity chamber at $80^{\circ} \mathrm{C}$ and $65 \%$ relative humidity for 10 minutes.

\section{$\underline{\mathrm{DFO}}[14]$}

DFO (0.5g, Sirchie) was completely dissolved in methanol (100mL, Fisher), ethyl acetate (100mL, Fisher) and acetic acid (20mL, Fisher) using a magnetic stirrer. Petroleum ether (780mL, Fisher) was then added with further stirring.

Samples to be treated with DFO were immersed in the solution for a few seconds, air dried and heated in a dry oven at $100^{\circ} \mathrm{C}$ for 20 minutes.

\section{Genipin [14]}

Genipin (1.71g, TimTec) was completely dissolved in ethanol $(57 \mathrm{~mL}$, Fisher) and ethyl acetate $(86 \mathrm{~mL}$, Fisher) using a magnetic stirrer. Once all the genipin was dissolved, petroleum ether $(857 \mathrm{~mL}$, Fisher) was added with further stirring.

As per ninhydrin, the paper sample to be treated was immersed in the solution for a few seconds. The samples were then allowed to air dry before being placed in a heat and humidity chamber at $80^{\circ} \mathrm{C}$ and $65 \%$ relative humidity for 10 minutes.

\section{Lawsone [14]}

Lawsone $(0.5 \mathrm{~g}$, Sigma) was dissolved in ethyl acetate $(100 \mathrm{~mL}$, Fisher) followed by the addition of petroleum ether $(400 \mathrm{~mL}$, Fisher) with stirring. As per genipin, the paper sample to be treated was immersed in the solution for a few seconds. The samples were then allowed to air dry before being placed in an oven at $150^{\circ} \mathrm{C}$ for 60 minutes. 


\section{Grading of fingermarks}

Following chemical enhancement, each fingermark was graded using the grading scheme recommended by CAST in table 3 [10].

Table 3: Grading scheme for assessment of developed as recommended CAST

\begin{tabular}{|c|c|}
\hline Grade & Level of Detail \\
\hline 0 & No evidence of mark \\
\hline 1 & Some evidence of contact but no ridge detail present \\
\hline 2 & Less than $1 / 3$ of mark showing clear ridge detail \\
\hline 3 & Between $1 / 3$ and $2 / 3$ of mark showing clear ridge detail \\
\hline 4 & Over $2 / 3$ of mark showing clear ridge detail \\
\hline
\end{tabular}

It should be noted that the grades attributed to each fingermark are, to some extent, subjective and other scoring methods currently being researched might provide different results. These grades were then accumulated into groups according to the enhancement reagent used and the ageing time of the fingermarks.

The split print method involves the comparison of techniques where a fingermark diminishing series is split into two prior to chemical treatment. The left side is then treated with for example genipin and the right side with ninhydrin, however the experiment is then repeated to treat the left side with ninhydrin and the right side with genipin. The process is complete once all the enhancement chemicals have been compared to each other. As four techniques were compared in this study, a total of six fingermarks were enhanced with genipin for every position in the diminishing series, for every paper substrate and for every ageing period. An average out of a total of six was calculated for fingermarks on $120 \mathrm{gsm}$ paper at position 1 in the depletion series' enhanced with genipin for a particular ageing period. To minimise the effect of variables that were present when planting the fingermarks, this was then repeated for all fingermark positions, all papers and all reagents.

The calculation of the average grading of the fingermarks minimised the effect of some of the variables that may have been present during the fingermark deposition stages. Such variables included the pressure used to plant each fingermark and variations in the temperature and consistency of the blood at the time of planting. The described methodology attempted to minimise the effect of these variables. 


\section{Results and Discussion}

\section{Positive and negative controls}

Positive control for the enhancement of latent fingermarks (without the presence of blood) was tested on all papers in the study. Ninhydrin resulted in purple-coloured fingermarks whereas DFO showed strong fluorescence but a limited visual magenta colour. Genipin and lawsone only revealed limited fluorescence but enhancement was poor overall. The negative control samples which contained no fingermarks (latent or in blood) revealed no colour or fluorescent enhancement.

\section{Genipin}

Genipin treatment of fingermarks in blood on all paper types showed no evidence of enhancement for all of the ageing periods, although some paper types appeared to exhibit background fluorescence improving the contrast with the fingermark. The genipin treatment was effective at the enhancement of some latent fingermarks where the substrates had been touched and handled. However, no enhancement of the ridges in blood was observed.

None of the genipin treated fingermarks displayed any fluorescence (figure 2). Genipin enhancement of fingermarks in blood on the $80 \mathrm{gsm}$ pink paper resulted in random speckled spots of fluorescence for the 4 week and 6 week samples (figure 3). These spots were contained mainly in the area of the fingermark but could also be seen outside this area for the 4 week old samples, displaying a different pattern of fluorescence than that of the background fluorescence. Previous research had demonstrated that genipin reacts with latent fingermarks that are composed mainly of eccrine sweat [2]. Some dyes or chemicals in the substrate may also have caused the fluorescent qualities of genipin to be quenched. 


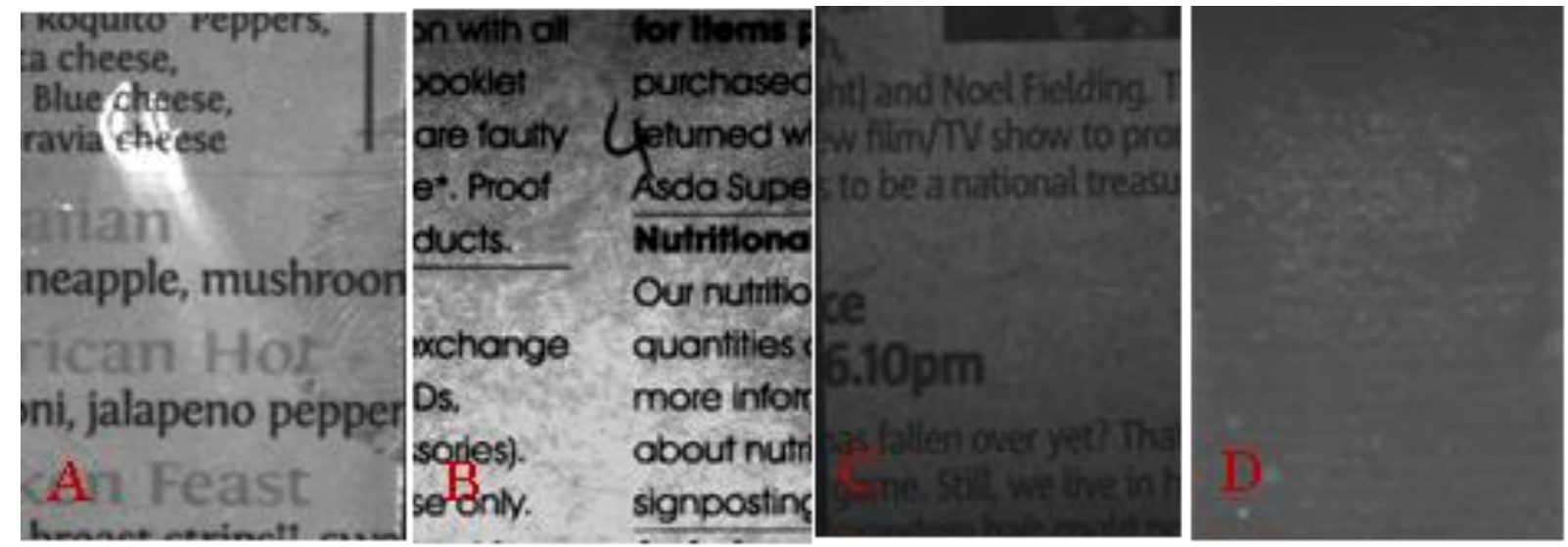

Figure 2: Fingermark in blood no 4 in the diminishing series on A) leaflet B) magazine C) newspaper and D) $120 \mathrm{gsm}$ paper after an ageing period of 1 week and treatment with genipin viewed using a 503-587nm excitation filter and a 593nm viewing filter

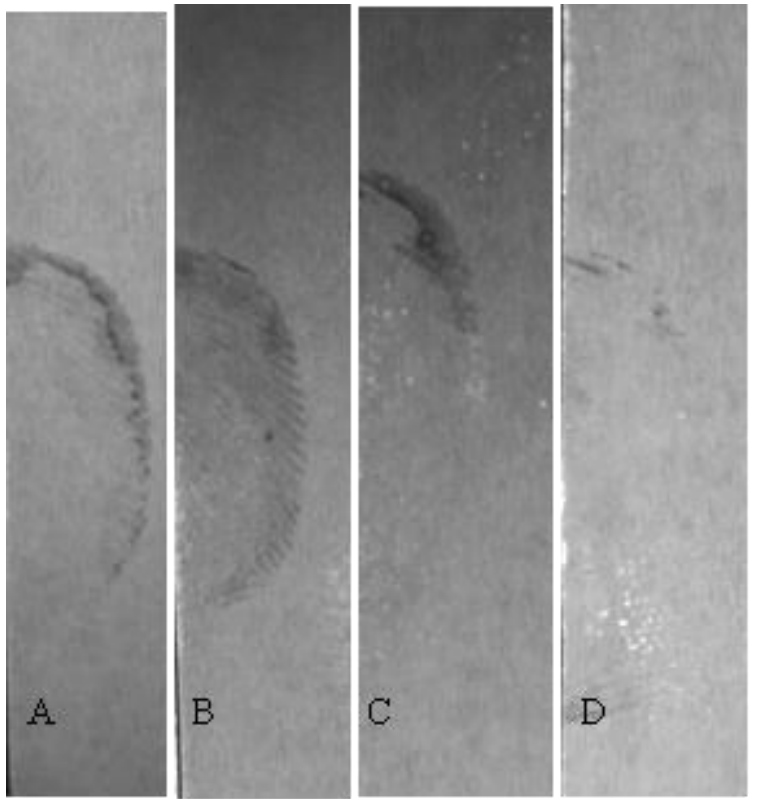

Figure 3: Genipin enhancement of fingermark in blood number 5 on pink paper after A) 1 week B) 2 weeks C) 4 weeks and D) 6 weeks, viewed using a 503-587nm excitation filter and a 593nm viewing filter 
During the grading of the fingermarks it was noticed that the genipin treatment appeared to be detrimental to the quality of some of the fingermarks. When left to age for 6 weeks, 5 fingermarks demonstrated a deterioration compared to before enhancement, the 4 week timescale showed 3 marks while the 2 week and 1 week ageing period showed 6 marks and 1 mark respectively.

\section{Lawsone}

The positive control for lawsone on latent fingermarks did not yield any enhancement (visual or fluorescent), in contradiction to other research which found that lawsone reacted with latent fingermark deposits to yield purple-brown ridge details with photoluminescent properties [4]. The reagent was prepared again with a fresh batch of lawsone but again no enhancement was observed. $98 \%$ of the fingermarks in blood treated with lawsone in this study demonstrated no enhancement, visual or fluorescent, as demonstrated in figure 4 . The detrimental effect that genipin had on some fingermarks in blood was also apparent for lawsone enhancement for fingermarks in blood deposited on 80 gsm paper and developed after 4 weeks and 6 weeks (figure 5). As with genipin, fluorescence quenching may have occurred due to dyes or chemicals present in the substrate.

The genipin and lawsone formulations in this study yielded limited enhancement with ridge detail diffusion. Diffusion for marks treated with other amino acid staining reagents, such as ninhydrin and DFO, has not been previously reported or observed in this study.

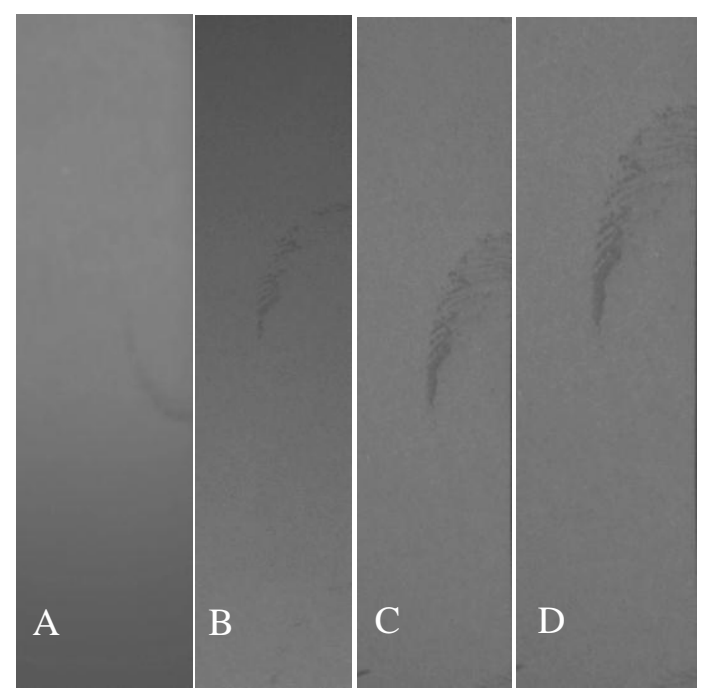

Figure 4: Lawsone enhancement of fingermark in blood number 5 on green paper after A) 1 week B) 2 weeks C) 4 weeks and D) 6 weeks, viewed using a 503-587nm excitation filter and a 593nm viewing filter 


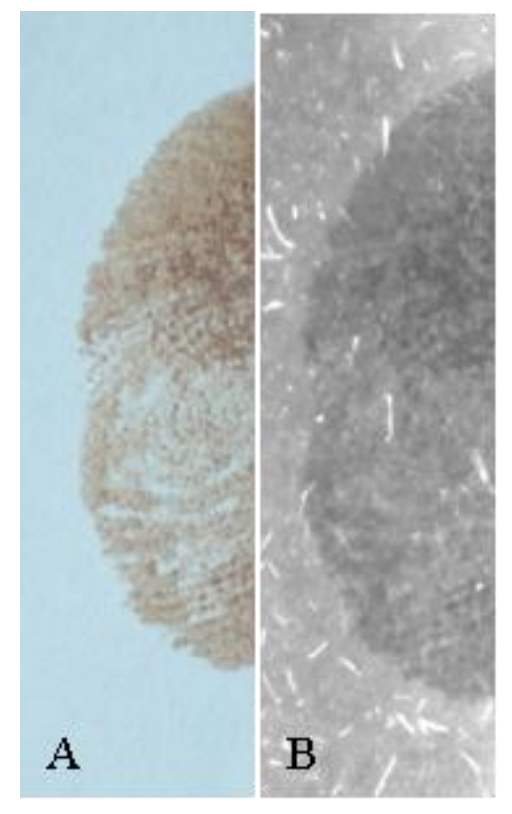

Figure 5: An example of a fingermark in blood treated with lawsone on $80 \mathrm{gsm}$ paper after a 6 week ageing period A) before development and B) fluorescence after development.

\section{$D F O$}

DFO showed some potential as a reagent for the development of blood contaminated fingermarks on paper. Figure 6 demonstrates that the enhancement of fingermarks in blood after an ageing period of 4 weeks exhibited some fluorescence and increased ridge detail visibility.
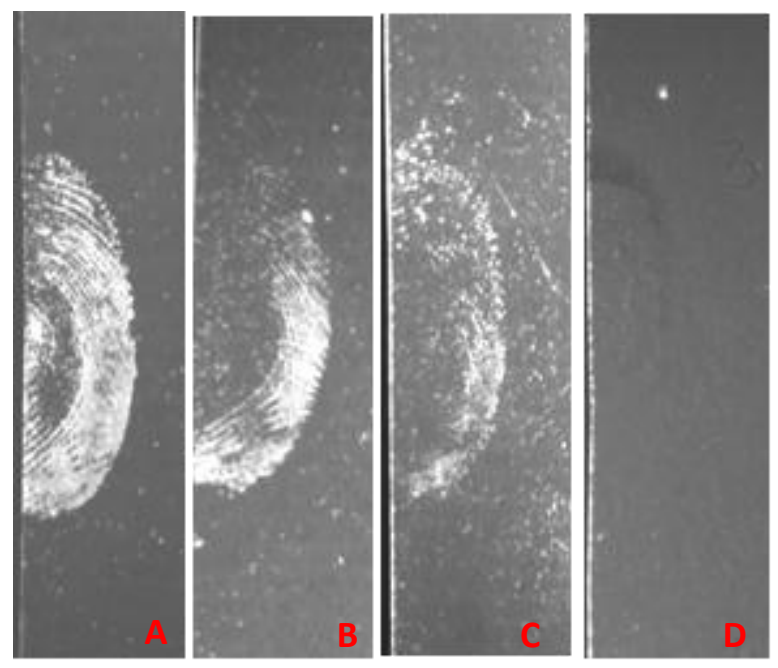

Figure 6: DFO enhancement of a fingermark in blood number 5 on A) magazine B) green C) 100 gsm and D) 120 gsm paper after a 4 week ageing period and viewed using a 491-548nm excitation filter and a 549nm viewing filter 
These fluorescent properties of DFO were only evident on the 4 week old fingermarks. This fluorescence was coupled with an increase in the quality and visibility of the ridge details evident on the fingermark. None of the other ageing periods demonstrated fluorescence and the DFO treatment mostly displayed a detrimental effect on the quality of the fingermarks. It has been suggested that the use of DFO for the enhancement of marks made in blood might be hindered by the nature of the substrate and associated fluorescence quenching where the enhancement of footwear impressions in blood on fabric with DFO was found to be ineffective [8]. Similar dyes and/or chemicals used in the manufacture of paper could be hindering the enhancement process leading to fluorescence quenching. It is also probable that the amines in the blood are reacting with DFO, however the fluorescence is then quenched by the haem groups present. As these haem groups in blood break down (due to ageing or heating), positive results may be more readily observed. Such quenching may also have occurred for genipin and lawsone (although the lack of any colouration may suggest a complete lack of reaction). This limited fluorescence appears to be contrary to other studies where DFO was found to be extremely sensitive for the development of fingermarks in blood on porous surfaces and also produced highly fluorescent marks with the lightest of the depleted marks [15].

Suitable enhancement was observed on 100 gsm paper, 80 gsm green papers and a magazine page suggesting that DFO may be capable of enhancing fingermarks in blood on varying paper porosities. It is also possible that the dyes used for colouring the paper may affect the enhancement of the blood contaminated fingermarks with DFO as both the green and pink papers had a porosity of $80 \mathrm{gsm}$ but only the green paper showed any evidence of fingermark development. A similar claim can also be said of the glossy properties of different papers as both the leaflet and the magazine displayed glossy surfaces but the fingermarks were only enhanced on the latter. 


\section{Ninhydrin}

Ninhydrin was successful in the enhancement of most depletion series' and also yielded clear ridge detail. The results demonstrated that ninhydrin was capable of enhancing fingermarks in blood on every paper type and colour with similar enhancement across the four ageing periods tested (figure 7). The fingermarks towards of the end of the depletion series' showed less enhancement than the marks at the beginning. This may suggest that the blood on the finger prevented the deposition of the latent component of the print as it was anticipated that enhancement would still be observed towards the end of the diminishing series due to fingermark secretions.

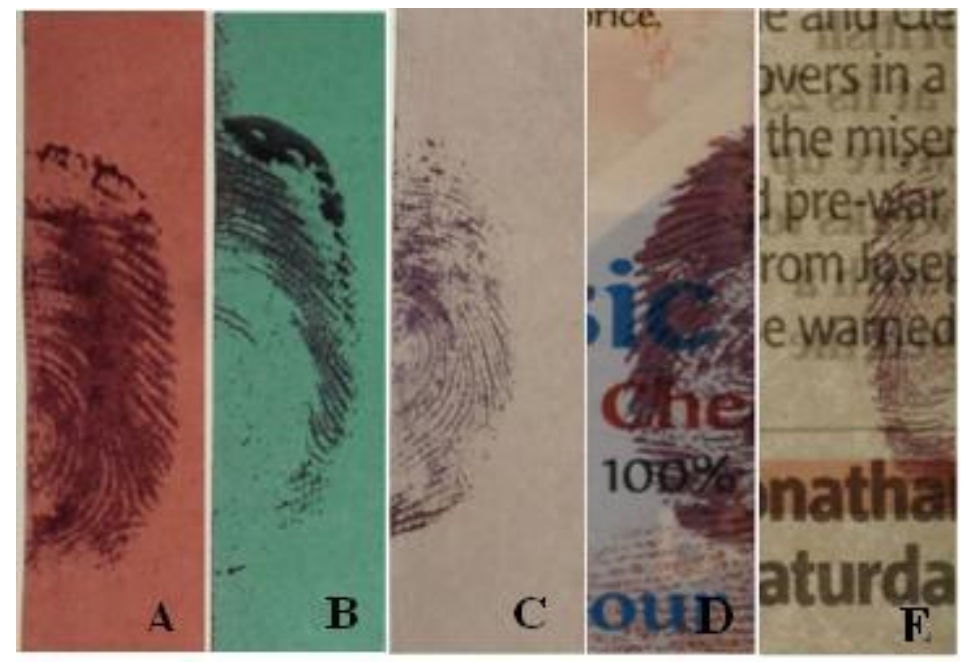

Figure 7: Ninhydrin enhancement of fingermark in blood number 5 on different types of paper: A) pink B) green C) 120 gsm D) leaflet and E) newspaper after a 2 week ageing period

The individual grading results revealed that ninhydrin provided superior enhancement of fingermarks in blood on the magazine and the takeaway leaflet. The successful enhancement of fingermarks in blood with ninhydrin across the four ageing periods suggests that ninhydrin can successfully enhance blood contaminated fingermarks that are either fresh or up to and at least 6 weeks old.

The fingermarks in blood prior to enhancement were all graded as 0 because although some blood was visible at the beginning of the diminishing series, no ridge detail was observed. This is mainly due to the methodology described where the dabbed finger in blood was pressed twice onto clean chemical-free blue paper towels to remove the excess blood prior to the deposition of the diminishing series. The results obtained revealed that the enhancement of fingermarks in blood with ninhydrin was superior to that provided by DFO, genipin and 
lawsone across all ageing periods (figure 8) and all paper substrates (figure 9) used in the study. These figures reveal the percentage of fingermarks that demonstrated some level of enhancement thus including any grading from 1 to 4 .

Prior to enhancement, all fingermarks in blood were graded as 0 as no ridge detail was visible. In most instances the enhancement grading for fingermarks in blood treated with genipin, lawsone and DFO was 0 as enhancement did not provide any improvement. Treatment with ninhydrin provided enhancement grading results ranging from 0 (enhancement did not provide any ridge detail therefore no improvement) to 4 (full ridge detail observed after enhancement) which appeared to be independent of the ageing periods used in this study (figure 10).

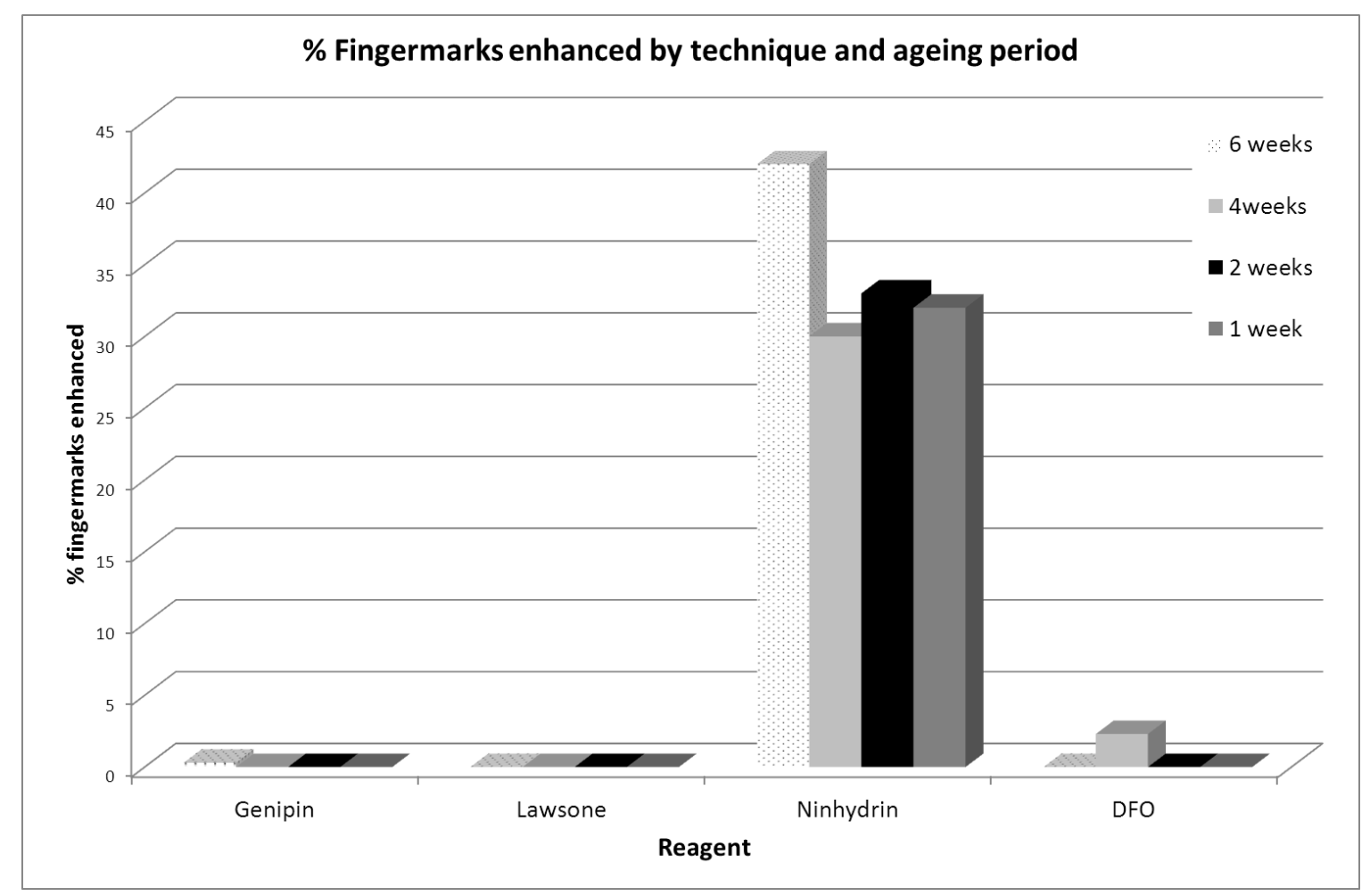

Figure 8 - Percentage of fingermarks in blood enhanced with genipin, lawsone, ninhydrin and DFO across the four ageing periods (grading ranging from 0 to 4 ) 


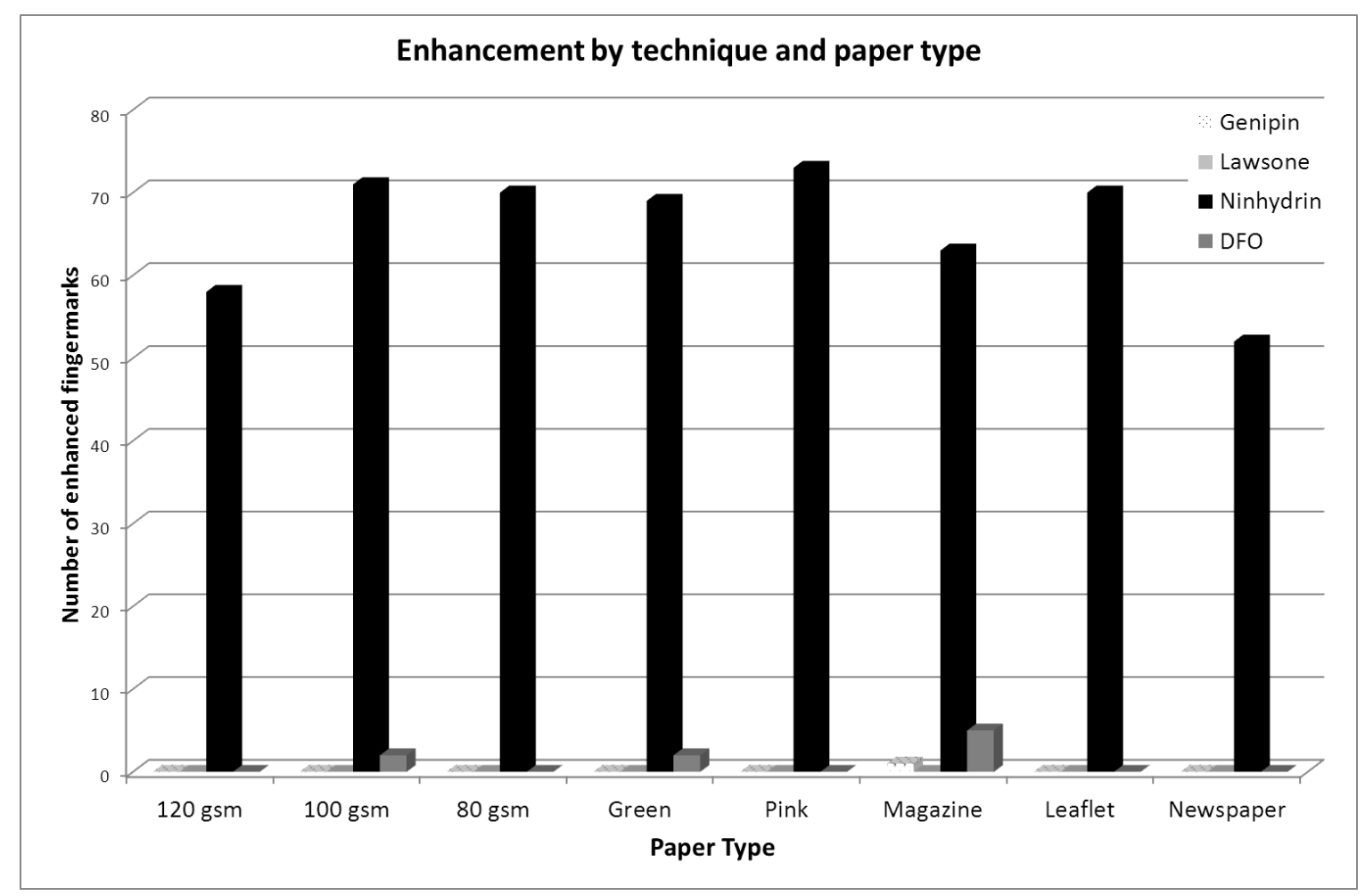

Figure 9 - Number of fingermarks in blood enhanced with genipin, lawsone, ninhydrin and DFO on all paper substrates in the study (grading ranging from 0 to 4)

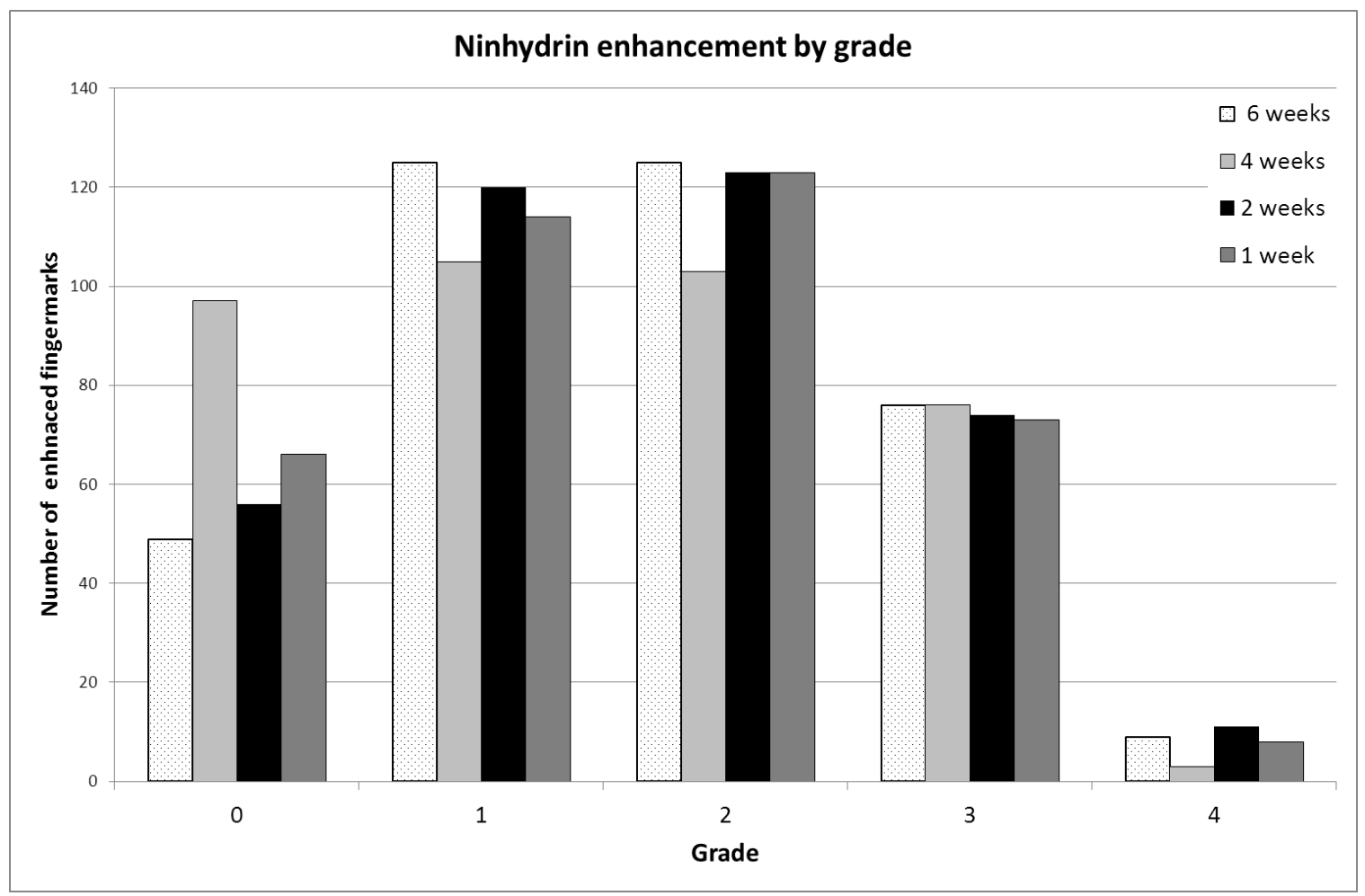

Figure 10 - Number of fingermarks in blood enhanced with ninhydrin on all paper substrates in the study (graded 0-4) 


\section{Conclusion}

This preliminary study showed no evidence to suggest that either genipin or lawsone are suitable reagents for the enhancement of blood contaminated fingermarks on different types of papers. In comparison to genipin and lawsone, ninhydrin appeared to be the most successful reagent for the enhancement of blood contaminated fingermarks on papers used in this study whereas DFO enhancement appeared to be limited. Fluorescence quenching and the limited reaction of the amino acids in blood with DFO, genipin and lawsone raises important questions and warrants further research in future work. While current research into other ninhydrin analogues or alternatives is beneficial and should be commended, it is likewise important not to shun away from 'well tried and document methods until reliable data and research is available' [16]. 


\section{References}

1. V. Bowman. 2005. Manual of Fingerprint Development Techniques. 2nd ed. Sandridge, UK: Home Office Scientific Development Branch.

2. J. Almog, Y. Cohen, M. Azoury, T.R. Hahn. Genipin - a novel fingerprint reagent with colorimetric and fluorogenic activity, Journal of Forensic Sciences, 49 (2) (2004) $255-257$

3. G. Levinton-Shamuilov, Y. Cohen, M. Azoury, A. Chaikovsky, J. Almog. Genipin: a novel fingerprint reagent with colorimetric and fluorogenic activity, part II: optimization, scope and limitations, Journal of Forensic Sciences, 50 (6) (2005) 1367 1371

4. R. Jelly, S.W. Lewis, C. Lennard, K.F. Lim, J. Almog. Lawsone: a novel reagent for the detection of latent fingermarks on paper surfaces, Chemical Communications, 30 (2008) 3513-3515.

5. R. Jelly, S.W. Lewis, C. Lennard, K.F. Lim, J. Almog. Substituted naphthoquinones as novel amino acid sensitive reagents for the detection of latent fingermarks on paper surfaces, Talanta 82 (5) (2010) 1717-1724.

6. Theeuwen, A.B.E.; van Barneveld, S.; Drok, J.W.; Keereweer, I.; Limborgh, J.C.M.; Naber, W.M.; Velders, T. Enhancement of Footwear Impressions In Blood, Forensic Science International, 95 (2) (1998) 133-151

7. R.A. Morgan-Smith, D.A. Elliot, H. Adam. Enhancement of aged shoeprints in blood, Journal of Forensic Identification, 59 (1) (2009) 45-58.

8. K.J. Farrugia, H.L. Bandey, K.A. Savage, N. Nic Daéid. Chemical Enhancement of Footwear Impressions in Blood on Fabric - Part 3: Amino Acid Staining, Science and Justice, 53 (1) (2013) 8-13

9. C. Stein, S.H. Kyeck, C. Henssge, DNA Typing of Fingerprint Reagent Treated Biological Stains, Journal of Forensic Sciences, 41 (6) (1996) 1012-1017

10. C.J. Fregeau, O. Germain, R.M. Fourney, Fingerprint Enhancement Revisited and the Effects of Blood Enhancement Chemicals on Subsequent Profiler Plus T Fluorescent Short Tandem Repeat DNA Analysis of Fresh and Aged Bloody Fingerprints, Journal of Forensic Sciences, 45 (2) (2000) 354-380

11. R. Jelly, E.L. Patton, C. Lennard, S.W. Lewis, K.F. Lim. The detection of latent fingermarks on porous surfaces using amino acid sensitive reagents: A review, Analytical Chemical Acta, 652 (1-2) (2009) 128-142 
12. V.G. Sears, S.M. Bleay, H.L. Bandey, V.J. Bowman. A methodology for fingermark research. Science and Justice, 51 (3) (2012) 145-16

13. S. Hardwick, T. Kent, V.G. Sears. Fingerprint Detection by Fluorescence Examination: A Guide to Operational Implementation. 1990, Home Office Police Scientific Development Branch 3/90: London.

14. R.S. Ramotowski. Amino Acid Reagents, in: Lee and Gaensslen's Advances in Fingerprint Technology, $3^{\text {rd }}$ Edition, R.S. Ramotowski, Ed., CRC Press: Florida, (2012) 17-54

15. V.G. Sears, C.P.G. Butcher, L.A. Fitzgerald, Enhancement of fingerprints in blood Part 3: reactive techniques, acid yellow 7, and process sequences, Journal of Forensic Identification, 55 (6) (2005) 741-763

16. T. Kent, Standardizing Protocols for Fingerprint Reagent Testing, Journal of Forensic Identification, 60 (3) (2010) 371-379 